(C) [2008] IEEE. Reprinted, with permission from [Li Zheng and Doan B. Hoang, Applying Graph Coloring in Resource Coordination for a High-density Wireless Environment, Proceedings of 2008 IEEE $8^{\text {th }}$ International Conference on Computer and Information Technology, CIT 2008]. This material is posted here with permission of the IEEE. Such permission of the IEEE does not in any way imply IEEE endorsement of any of the University of Technology, Sydney's products or services. Internal or personal use of this material is permitted. However, permission to reprint/republish this material for advertising or promotional purposes or for creating new collective works for resale or redistribution must be obtained from the IEEE by writing to pubs-permissions@ieee.org. By choosing to view this document, you agree to all provisions of the copyright laws protecting it 


\title{
Applying Graph Coloring in Resource Coordination for a High-density Wireless Environment
}

\author{
Li Zheng and Doan B. Hoang \\ iNEXT - UTS Centre for Innovation in IT Services and Applications, Faculty of Information Technology, \\ University of Technology, Sydney, NSW 2007, AUSTRALIA. \\ 1.zheng@ieee.org, dhoang@it.uts.edu.au
}

\begin{abstract}
In a high density wireless environment, channel interference among users of many overlapped Basic Service Sets (OBSSs) is a serious problem. Our solution for the problem relies on a resource coordination scheme that utilizes the spatial distribution of the transceivers for channel reuse and time-slot division multiplexing for downlink transmission sharing among all participating BSSS. In this paper we show that an OBSS environment can be modeled by a planar graph and the OBSS group coordination assignment problem can be considered as a vertex coloring problem whose solution involves at most four colors. The graph coloring solution algorithm for the OBSS group coordination assignment is presented. The actual coloring is demonstrated, using a heuristics of Maximum Degree First. Performance simulation results of the coordination algorithm are also presented.
\end{abstract}

\section{INTRODUCTION}

high-density packet radio wireless network is a $\mathbf{X}_{\text {collection of radio transmitters and receivers }}$ (transceivers) located in a close geographical region. Associated with each transceiver is a transmission range, which depends on its transmission power. A transceiver client $_{a p 1,1}$ located within the transmission range of another

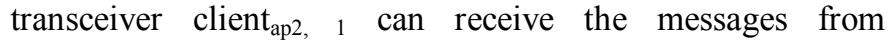
transceiver client $t_{a p 2,1}$. The transceivers of the network share the communication medium for message transfer. This gives rise to conflict situations where two or more of the transceivers may want the same shared medium simultaneously. In a coordinated OBSS environment, it means the sharing of a common radio channel for all BSSs (and all its clients) without causing garbling and eventual loss of messages.

The spatial distribution of the transceivers permits sharing or reuse of the channel. Most popular techniques for this sharing includes time slot division; frequency division and code division multiple access schemes. The medium division or scheduling are often modeled and solved by the graph coloring technique, where the radio network is modeled using graph. In our overlapping resource coordination scheme, the medium is scheduled for sharing among all participating BSSs by time slot division technique. In this paper, we investigate the nature of overlapping BSS environment and provide a mapping between the OBSS group assignment and the graph coloring problem. We demonstrate that the solution to the coloring problem is in fact an efficient solution for OBSS group coordination and assignment for avoiding interferences. The rest of the paper is organized as follows. Section 2 outlines the resource coordination scheme, where group assignment plays an important role. Section 3 discusses how to map the group assignment to graph coloring problems. Section 4 studies the NP-completeness of the coloring solution. Finally, we present the actual procedure of the colouring and its performances in Section 5 and 6.

\section{OBSS RESOURCES COORDINATION}

All BSSs in such coordinated OBSS environment would be synchronized and have a unique and coordinated superframe structure. Each coordinated BSS simultaneously operates in either downlink polling period or uplink contention period. In our OBSS downlink channel assignment solution, the channel sharing is based upon time, spatial reuse is to have users (BSSs) sufficiently far apart (but in a reuse group) to use the same frequency band. Members in the same reuse group are generally interference-free to each other and have certain physical distance sufficiently far apart from each other. Neighboring BSSs, which are in different groups, could not be assigned the same channel simultaneously. Therefore, the downlink period is divided into several limited time slot/span for these reuse groups. Apparently, the less number of time slots are divided, the more efficient the channel assignment solution is. In this paper, we focus on the details of channel assignment for downlink transmission only.

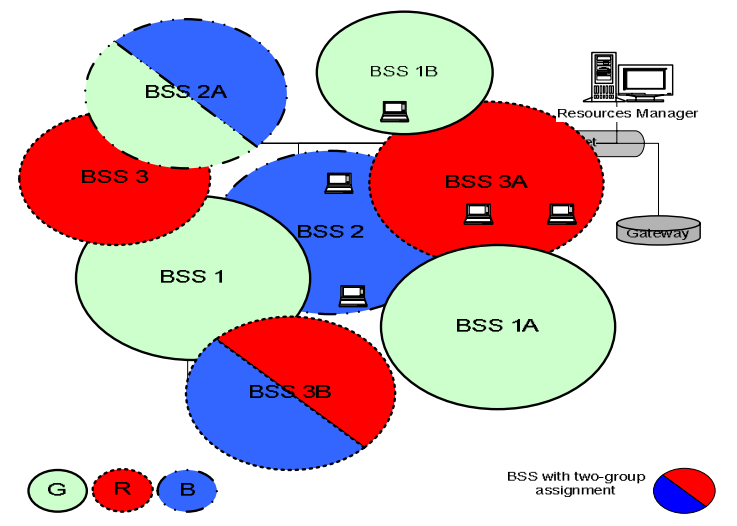

Figure 1 An Example of the First and Second Group Assignment

The purpose of channel (group) assignment computation is to produce guidelines for dividing all participating BSSs into several re-use groups, namely Group R (Red), B (Blue), G (Green), Y (Yellow), etc. The constraint of grouping is that no two overlapping BSSs could be assigned into the same group. The objective of grouping is to find the minimum number of groups for complete coverage. The decision purely depends on the number of BSSs in the coordinated environment and the 
overlapping severity (the neighboring/overlapping relationship among all participating BSS). In order to maximize the spatial reuse, we propose second grouping assignment, which BSSs with high downlink traffic may be additionally assigned its second group to satisfy their demands. The second grouping neither increases the number of groups used nor changes the result of the first grouping assignment. Instead, it only assigns additional group to certain BSSs. The constraint of the second grouping remains the same as that in the first grouping, which is every two overlapping BSSs, could not be assigned into the same group.

In such ways, each BSS is at least assigned one group and this group contains such BSSs members that do not overlap and interfere with each other. A simple example of an 8 colocated BSSs environment is given and the results of the first and second grouping for this example are shown in Figure 1. All BSSs are assigned into 3 groups of R (BSS 3, 3A, 3B), B $(2,2 \mathrm{~A})$ and $\mathrm{G}(1,1 \mathrm{~A}, 1 \mathrm{~B})$; while $\mathrm{BSS} 2 \mathrm{~A}$ and $3 \mathrm{~B}$ are assigned with two groups. As seen, BSSs in the same group/color are not overlapped and interference-free. And only three colors are used for the assignment. We work through details of grouping using graph coloring with this example as below.

\section{MAPPING TO GRAPH THEORETIC PROBLEM}

Due to the similarity between scheduling and some graph theoretic problem, we solve our OBSS group assignment problem using graph theory; therefore, the problem could be considered in a simpler way and certain theorems and techniques in graph theories could be used to solve the problem. If it could be modeled as a graph with a vertex for each cell and edges to represent interference, this group assignment / scheduling problem can be solved using graph vertex coloring technique.

\section{A. Basic Graphical model for Channel Assignment}

The channel assignment problem could be mathematically modeled in a few ways. The most famous models are multiple interference model and binary constraint model [1]. The multiple interference modeling studies the signal propagation, defines attenuation factors for adjacent channel interference and evaluates Signal-to-Interference Radio at certain test point in order to assign channel properly without occurrence of interference. In the binary constraint model, constraints are only binary over pairs of variables (instead of three or more). Therefore, it has a higher level of abstraction and requires less computational complexity. It can be seen as a generalization of the well-known Graph Coloring Problem. In this paper, we adopt this model and focus on how the OBSS channel assignment could be modeled as a Graph Coloring Problem.

Our presentation of channel assignment is based on a standard representation of a radio network by a directed graph $\mathrm{G}=(\boldsymbol{V}, \boldsymbol{E})$. We limit that graph only refers to a network of edges and vertices. Here, $\boldsymbol{V}$ is a set of vertex denoting the clients in the radio network, and $\boldsymbol{E}$ is a set of directed edges between vertices such that for any two distinct vertices $\boldsymbol{u}, \boldsymbol{v} \in$ $\boldsymbol{V},(\boldsymbol{u}, \boldsymbol{v}) \in \boldsymbol{E}$ if and only if $\boldsymbol{v}$ can receive $\boldsymbol{u}$ 's transmission. In such way, $G$ denotes an interference graph, where the node set denotes cells or base stations (BSS) that require communication service, and the edge set represents geographical approximation of cells and therefore the possibility of co-channel interference.

Above, the standard definition of the edges is with directedness. That is, $(\boldsymbol{u}, \boldsymbol{v}) \in \boldsymbol{E}$ does not imply that $(\boldsymbol{v}, \boldsymbol{u}) \in \boldsymbol{E}$. In fact, within WLAN environment, even though the interference between cells may not be bi-directional, the information necessary to make assignment decision is the fact that interference occurs, no matter which transceiver of two adjacent transceivers causes the interference to occur. And such information is sufficient to schedule the channel assignment to prevent interference. Therefore, from the assignment point of view, the network can be represented by an undirected graph. That is, if there is/are $(\boldsymbol{u}, \boldsymbol{v}) \in \boldsymbol{E}$ or / and $(\boldsymbol{v}, \boldsymbol{u}) \in \boldsymbol{E}$, we establish the undirected edge between $\boldsymbol{u}$ and $\boldsymbol{v}$.

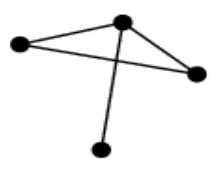

Simple Gragh

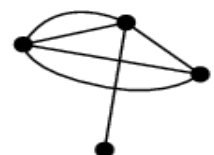

Multi-gragh

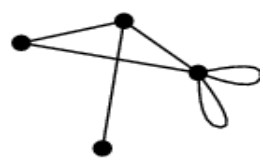

Pseudo-gragh
Figure 2 Graph Varieties

Graphs also come in a wide variety of different sorts as shown in Figure 2. The most common type is graphs in which at most one edge (i.e., one edge or no edge) may connect any two vertices are said to be simple graphs. If multiple edges are allowed between vertices, the graph is known as a multigraph. Vertices are usually not allowed to be self-connected, but this restriction is sometimes relaxed to allow such "graph loops." A graph that may contain multiple edges and graph loops is called a pseudo-graph. A simple graph is sufficient enough to represent the wireless network model. Therefore, in our graph, there are no multiple edges and graph loops. Further formal definitions of terms we use through this section are as follows. If $(\boldsymbol{u}, \boldsymbol{v}) \in \boldsymbol{E}$, then $\boldsymbol{v}$ is a neighbour of $\boldsymbol{u}$. As our graph is undirected, $\boldsymbol{u}$ is also a neighbour of $\boldsymbol{v}$. A neighbour must be a vertex. The degree of a vertex $v$ is the total number of neighbours of $\boldsymbol{v}$. The maximum degree of a graph is the maximum of degrees taken over all vertices in the simple graph.

\section{B. Corresponding Graph Coloring and its Constraint}

The edges, vertices, or both of a simple graph may be assigned specific values, labels, or colors (properties), in which case the graph is called a labeled/colored graph. A vertex coloring is an assignment of labels or colors to each vertex of a graph such that no edge connects two identically colored vertices. Similarly, an edge coloring is an assignment of labels or colors to each edge of a graph such that adjacent edges (or the edges bounding different regions) must receive different colors. The assignment of labels or colors to the edges or vertices of a graph based on a set of specified criteria (constraints) is known as graph coloring. A constraint is defined as a symmetric relation between two vertices or two 
edges in a graph. It imposes a restriction on coloring: two vertices or edges that are mutually constrained (related by some constraint) must receive different color for an assignment to be legal. So it is normally described by whether they are between vertices or edges, the separation between them, and whether it is a transmitter and/or a receiver based constraint. It is either a vertex based or an edge based coloring.

The OBSS group assignment problem models the task of assigning time slot to a set of Overlapping BSSs. A feasible group assignment must additionally satisfy certain interference constraints. An optimal assignment globally minimises a cost function, which is the minimum of time slots it uses in the OBSS system. It is important to understand that interference can only be defined with respect to a receiver. That is, any signal, occurring within the same channel that a desired signal is to be received in, is considered to interfere with the reception of the desired signal if it is of sufficient power (as measured at the designated receiver). Interference power is a function of transmitter power, receiver sensitivity, antenna gains, patterns and polarizations, and channel loss. Channel loss is a function primarily of distance, frequency, and weather and is quantified by minimum acceptable signal to noise power ratio, or maximum permissible interference to noise power ratio as measured at a receiver. We can assume that most of these factors are already determined or beyond our ability to influence. Consequently, we will define interference directly as a function of distance in the case of OBSS problem.

Furthermore, during downlink transmission period, only those cells (vertexes), which could hear other (BSS is detected by either or both sides), would cause interference between themselves. Therefore, such constraint for OBSS group assignment is vertex based. And these cells are often colocated and adjacent, so called neighbours. The interference will not be carried out further to neighbour's neighbour as long as there is no overlapping detention among them. The constraint is so called Distance-1. And it makes no difference whether constraint is transmitter and/or a receiver based, with the same reason that undirected graph is applied here. Distance-1 graph vertex coloring of an undirected graph is defined for the OBSS group assignment problem. In a simple way, all adjacent vertexes/BSSs should not receive the same label/color. In the mathematics way, given an undirected graph $\mathrm{G}=(\boldsymbol{V}, \boldsymbol{E})$, a Distance-1 graph vertex coloring of an undirected graph, is a mapping -

$$
\left\langle f(v) \rightarrow N \mid f\left(v_{i}\right)=f\left(v_{j}\right) \Leftrightarrow\left(v_{i}, v_{j}\right) \notin E\right\rangle .
$$

\section{Representing Graphs and its Properties}

The selection of graph representation for a network should give consideration to the characteristics of the network scenario, which is modelled by the particular type of graphs. Model graphs are then selected designed to encapsulate the essential features of the system. The discussion in the previous study [3] [4] [5] clearly shows that, almost all prior research in this area used an arbitrary graph as the model of the radio network. Arbitrary graphs have the advantage of being able to represent all possible network configurations. And certain restricted graphs could give an accurate representation to certain radio network or network scenario; and illuminate some aspects of the problem structure, which might help in solving the problem, and finding the optimal solution, such as finding a chromatic number. Tree and planar graphs are most famous restricted arbitrary graphs used in modeling radio networks. Tree is the simplest graphical representation and problems such as message routing and propagation can be well addressed using tree models. However, the tree structure is not flexible enough to represent many possible network configurations. The limitation of trees led Ramanathan to propose planar graphs as the model of choice [2] [3].

A planar graph is a graph that can be drawn in a plane without graph edges crossing. A planar graph (directed or undirected) can model a radio network for capturing essential features of a WLAN network [2]. For radio networks, BSSs can be considered as points on a two-dimensional plane. Associated with each node/BSS is a range value, representing the transmission range of the BSS. A graph can then be constructed where each node/BSS represents a point on the plane. An undirected edge connects node $\mathrm{Vi}$ to node $\mathrm{Vj}$ if the BSSi detects the presence of the BSSj or the BSSj detects the presence of the BSSi. The planar graph constructed in this manner captures all the pertinent features of the WLAN OBSS radio network.

However, when considering nodes as transmitter or transceiver, not only is the direction of interference considered, but also the geometric node (network) configuration is carefully concerned. Unlike most scenarios, the OBSS environment is defined by the location of each BSSs and the respective detection of other BSSs from such BSS. Considering the practical deployment scenario, the amount of BSS deployed should not be as large as that of transmitter or transceiver station in a normal radio network and the distance among neighbouring BSSs (nodes) should not be as close as those in a normal radio network. The OBSS environment should therefore be relatively planar, with a possible light network density in term of BSS number.

On the other hand, when there are many nodes deployed closely and overlapped heavily with each other, these nodes potentially cause dense interference and these are reflected as large number of edges within a limited area on the graph. Such network could only be represented by a planar point graph as shown in Figure 3, as the edge crossing could not be avoided. However, in practice, a poor planar deployment of BSSs is unlikely as discussed. Furthermore, the extreme limitation on resource within such small physical scope would not allow efficient communication for all these closely-located BSSs. Therefore, a planar graph is more appropriate to represent OBSS environment illuminating some aspects of the problem structure and helping in solving the problem (finding 
the chromatic number).
Tree

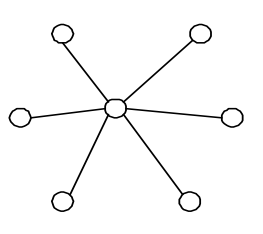

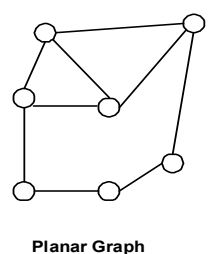

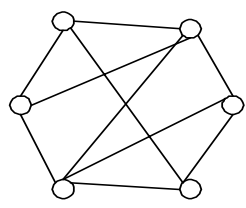

Planar Point Graph
Figure 3 Graphs Used for Modeling

\section{The Problem Solution And Completeness OF CHROMATIC NUMBER}

The most common type of vertex coloring seeks to minimize the number of colors for a given graph. Finding a minimal coloring can be done possibly using brute-force search [6]. The minimum number of colors, which with the vertices of a graph $G$ may be colored is called the chromatic number, denoted $\boldsymbol{X}(\boldsymbol{G})$. In general, a graph with chromatic number $n$ is said to be an $n$-chromatic graph, and a graph with chromatic number, which is less than or equal to $n$, is said to be $n$ colorable. Calculating the chromatic number of a graph is an $\mathrm{NP}$-complete problem. If a problem is known to be NPcomplete (Nondeterministic Polynomial), it can be guessed and verified in polynomial time. Even no particular rule is followed to make the guess when solving the problem, the solution obtained using a polynomial algorithm would not necessarily be optimal but reasonably close. Previous graph studies [3] [6] [7] have proved the NP-completeness according to most different types of graph.

Mapping to the OBSS group assignment, the Distance-1 graph vertex coloring technique is applied to find the minimum colors / re-use group needed for the OBSS group division. If a planar graph is used for modelling the network, it is an at least NP-complete problem [7] [3] and the four-color theorem could be applied. The four-color theorem states that any map in a plane can be colored using maximum four colors in such a way that regions sharing a common boundary (other than a single point) do not share the same color. More specifically, NP-completeness of a planar graph problem is proved, and the chromatic number for the assignment problem where the network can be modelled using the planar graph is 4. The chromatic number should remain 4 as long as the network could be represented by a planar graph regardless of number of nodes/BSSs.

However, NP-completeness of a planar point graph is not necessarily true; even it is a restricted class of the planar graph. Obviously, as network density increases, planar point graphs may be used instead of planar graph, most likely more colors need to be applied. At the mean time, the network efficiency is concerned. This should be considered particularly before deploying OBSS. When deploying the network, we should follow an engineering limit of a 'maximum' network density, in order to maintain network efficiency and ensure four-color theorem applicable (avoiding planar point graph). In [8], it is proved that as a fundamental property of a planar graph, the minimum degree vertex has at most five neighbours. In other words, it could be used to examine the 'maximum' network density when deploying the OBSS and trying to avoid over-dense situation. Therefore, guideline for not being over-dense is that:

\section{The BSS, which has minimum neighbours, has at most five neighbors.}

In the engineering practice, this could be done by relocating the BSS or limiting its transmission range. In such, the OBSS environment could be modeled by a planar graph, maximum four time spans could be sufficient in scheduling the downlink traffic without collision.

\section{The Colouring and its Performances}

\section{A. The Procedure and Coloring Results}

We now consider how to perform this Distance-1 undirected vertex coloring in a planar graph, which could model the common OBSS environment. There are mainly two classes of algorithms: geometric algorithms (for a 'linear' network) and greedy algorithms [3]. The greedy algorithm is chosen here because it has wider applicability to most graphs, including planar graph and its vertex coloring. The greedy algorithm mainly assigns the first non-conflicting color based on the colors already assigned to nodes. It consists of two phases, a labelling phase and a coloring phase. In the labelling phase, each vertex in the graph is firstly assigned a unique label between 1 and $n$, where $\mathrm{n}$ is the total number of the vertices. The vertices are then considered in decreased order of labels to be colored in the coloring phase. The coloring is done until the vertex labeled 1 is colored. The color assignment follows the greedy principles that no constrains is violated. As seen, the labelling determines the coloring order and largely determines the final results. There are a few possible ordering, such as the random ordering, Maximum Degree First (MDF) and Maximum Degree Last [9]. On average, most greedy algorithms produce similar results closer to optimal. The random ordering is the simplest and straightforward, so called the Pure Greedy. Even MDF presents slightly complex ordering technique, which results in higher time complex, its performances begin to diverge as the density (node population) of network increase. Therefore, MDF is chosen in this paper. The section below depicts the procedure of Maximum Degree First with an example shown in Figure 1.

The Maximum Degree First ordering (MDF) is also called Minimum Neighbour First. Here, degree is defined as the number of neighbours for this vertex. In this method we take a maximal mutually conflicting clique of edges around the maximum degree vertex first, color it, and then progressively do the same for the remainder of the graph. This method is based on the intuitive notion that it is better to color the more 'crowded' areas first. This ordering heuristic is based on the degree of each node. The determination of the degree is dynamic and the order is not determined, prior to any node being colored. In fact, a node with maximum degree is selected and colored and the degree of all its adjacent nodes 
are decreased before the next node is selected and colored. In the coloring phase, it would follow the principle of "firstavailable-color', where colors are given priorities to be selected without collision. Below, the first group assignment technique, using Maximum Degree First algorithm, is also given in formal specification. As seen, Procedure of AssignLabel (G) works out the degree of each BSS; and Procedure of First-Available-Color $(\mathrm{G}, v, \mathrm{C})$ colors the chosen BSS and keep the first avaliable color for the next loop.

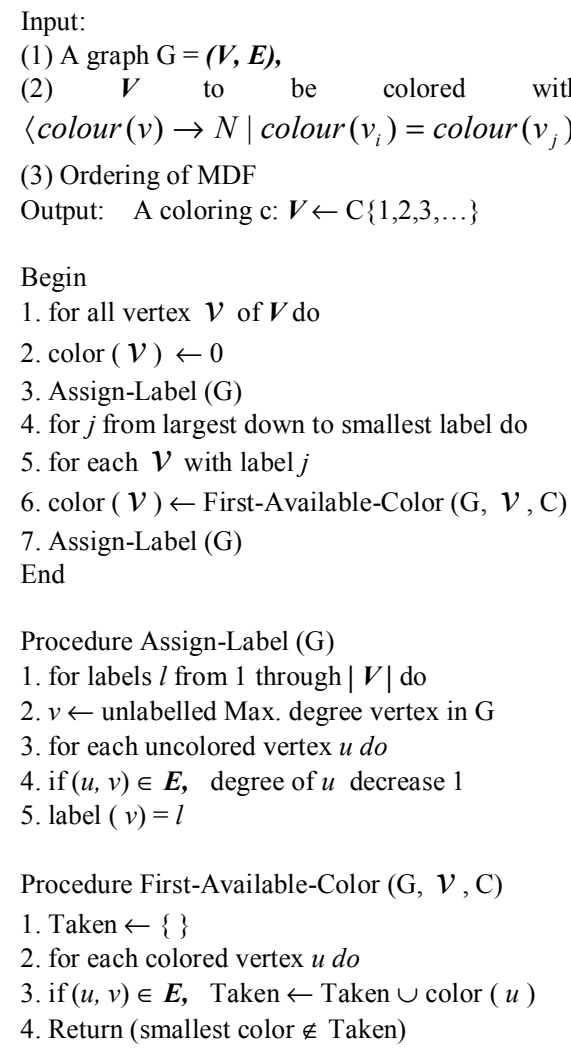

The second group assignment technique, using Maximum Degree First algorithm, is given in formal specification as below:

\section{Input:}

(1) A graph $\mathrm{G}=(\boldsymbol{V}, \boldsymbol{E})$,

(2) Every vertex $\boldsymbol{V}$ of $\boldsymbol{V}$ is assigned one color $(\boldsymbol{V} \leftarrow \operatorname{Color}\{1,2,3, \ldots\}$ and to be assigned second color with constraint of $\langle$ colour $(v) \rightarrow N|$ colour $\left(v_{i}\right)=$ colour $\left.\quad\left(v_{j}\right) \Leftrightarrow\left(v_{i}, v_{j}\right) \notin E\right\rangle$ (3) $\boldsymbol{V} \leftarrow$ Downlink Demand $\{1,2,3, \ldots\}$

Output: A coloring c: $\boldsymbol{V} \leftarrow \mathrm{C}\{1,2,3, \ldots\}$

Begin

1. for $j$ from largest down to smallest Demand do

2. for each $v$ with label $j$

3. Second-Color $(\mathcal{V}) \leftarrow$ First-Available-Second-Color $(\mathrm{G}, \quad \mathcal{v}, \mathrm{C})$ End

Procedure First-Available-Second-Color (G, $v, \mathrm{C})$

1. Taken $\leftarrow\{\}$

2. for each colored vertex $u$ do

3. if $(u, v) \in \boldsymbol{E}$, Taken $\leftarrow$ Taken $\cup$ color $(u)$

4. Return (smallest color $\notin$ Taken)
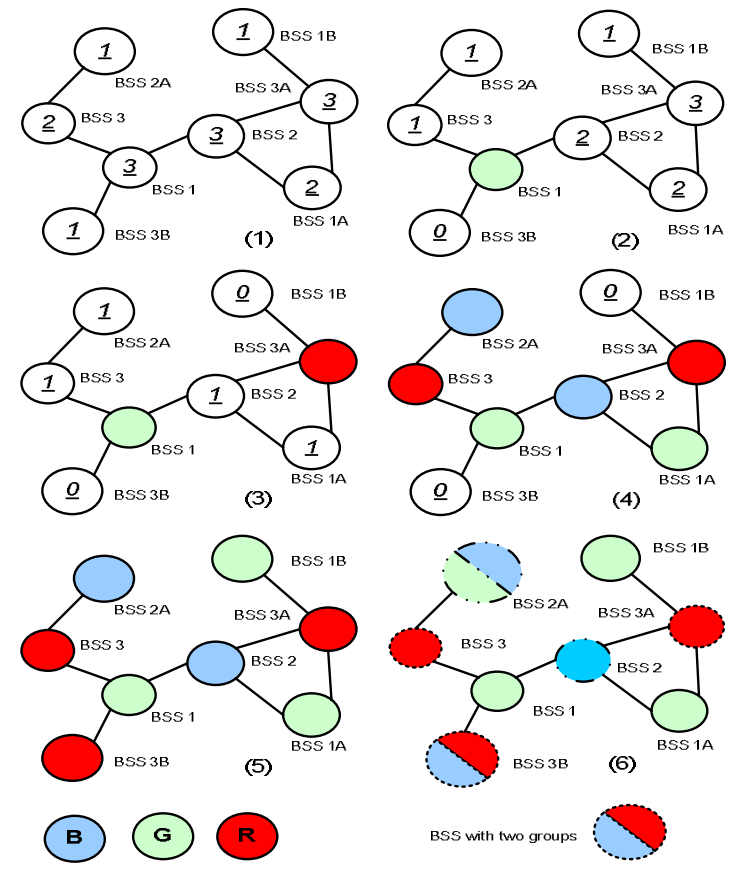

Figure 4 Example of Maximum Degree First on a Planer Graph

We work out the MDF procedure with an example planar graph as below. (1) of Figure 4 shows us the planar graph model for network shown in Figure 1. The degree of each BSS is shown within the circle and updated by each step. We firstly color the BSS 1 with Green and determine the degree for the rest of BSSs in (2). As seen, BSS 3A has the maximum degree of 3 and is selected firstly for coloring with Red. As BSS 2, $1 \mathrm{~A}, 1 \mathrm{~B}$ are all adjacent to BSS $3 \mathrm{~A}$, their degrees are then recalculated (decreased by 1) and shown in (3). BSS 1A, 2, 3, 2A now have degree of 1 . So the coloring for these four BSSs is in a random order. Particularly, when coloring BSS 1, it follows the principle of 'first-available-color' and only Blue is able to be used as Red and Green are not avaliable. (5) finally shows us the result of first grouping. As BSS 2A and 3B are busy BSSs, they have requests for second group assignment. Again, we follow the 'first-available-color' while remaining the result of the first grouping as in (5); BSS 2A is then assigned with Green as the second color and BSS 3B has Blue. The final result of first and second grouping is shown in (6). It has also shown us that this simple planar graph is 3 -colorable.

\section{B. The Coordination Performances}

Having the inputs of the network topology, RM could always divide all BSSs into maximum four groups, using the graph coloring algorithm as above. Therefore, during the downlink transmission, the OBSS interference could always be prevented and medium utilization could be achieved. Below, we present some preliminary results showing the performance of our resources coordination and graph coloring in terms of traffic QoS, medium utilization and fairness among the participated BSSs. Here only downlink performances are discussed, while uplink performances and other recommendations would be further studied in another paper. 
IEEE 802.11 WLAN OBSS environment is simulated using OPNET with standard simulation attribute values. The network configuration in the downlink simulation consists of the BSS 1, 2, and 3B as the group representatives, which colocate and overlap with each other as shown in Figure 1. Each BSS would only have downlink traffic and its AP simultaneously transmits multiple applications (VoIP, light Video Conference (VC), Audio and bulk data) to its clients at all time. BSS 1 has three VoIP transmissions; BSS 2 houses light $\mathrm{VC}$ and Audio; and BSS 3B is overloaded with large bulk data at $3.68 \mathrm{Mpbs}$ mean arrival rate.

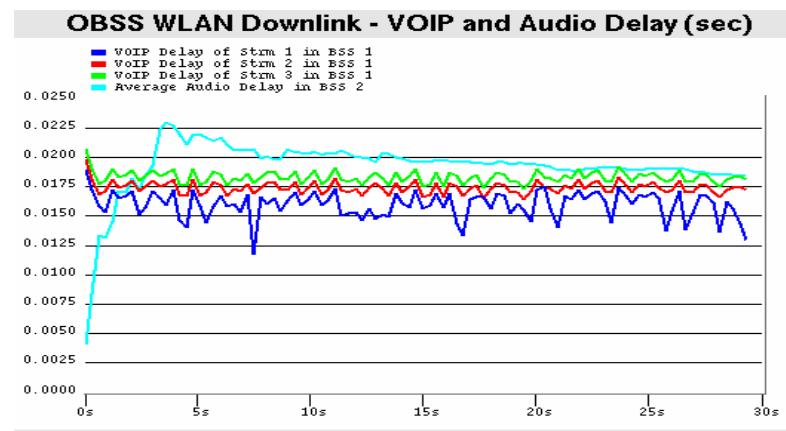

Figure 5 Delay for VoIP and Audio in BSS 1and 2 of Downlink Scenario

As seen in Figures 5, three VoIPs in BSS 1 have good delay performances, which all are less than 20ms. BSS 2 also maintains a satisfied performance for an audio and light VC, as its audio stream performs at less than $25 \mathrm{~ms}$ delay. The BSSs do not experience interference with satisfied performances as the result of grouping. On the other hand, bulk data in BSS 3B experiences a data drop at $1.75 \mathrm{Mbps}$. The data application reaches the capacity of BSS $3 \mathrm{~B}$ during the assigned duration and achieved a throughput of $1.8 \mathrm{Mbps}$ only as shown in Figure 6 (blue line). While BSS 3B is overloaded with such aggressive bulk data and has consequent data drop, it could not gain further transmission opportunities and make any impact on other applications from the co-located BSS 1 and 2. It demonstrates fairness among the co-located BSSs.

Figure 6 also shows the efficiency improvement in term of bulk data throughput in busy BSS 3B, gained by using the additional second grouping assignment. The blue line shows us a throughput of $1.8 \mathrm{Mbps}$ only and bulk data experiences the drop. Due to overloading on BSS 3B, RM performs the second group assignment and allow BSS 3B has additional second time slot, which enable a full throughput of $3.68 \mathrm{Mpbs}$ in BSS $3 \mathrm{~B}$ as shown on Figure 6.

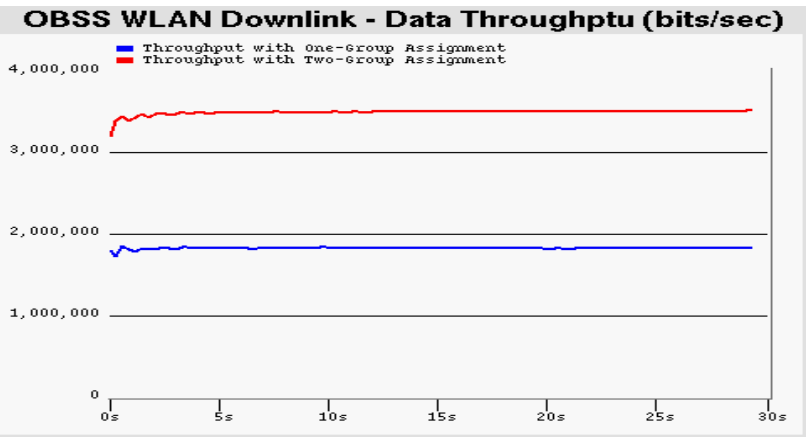

Figure 6 Bulk Data Throughput in BSS 3B of Downlink Scenario for First and Second Group Assignments

\section{Conclusions}

We have illustrated that an OBSS environment can be modeled as a planar graph in most practical cases. The OBSS channel assignment problem can be considered as a Distance1 undirected vertex coloring problem on such graphs. This vertex coloring problem has been proved NP-completeness as long as it is a common planar graph. In other words, maximum 4-color/time span is sufficient regardless of the sizes of network and number of BSSs involved, as long as the network is not over-dense (modeled as planar point graph). We have also provided a deployment guideline to avoid over-density situation (so to ensure four-color theorem applicability) to prevent network inefficiency. A coloring example of a planar graph with 3 colors, using a heuristics of Maximum Degree First, has also been given. Finally performances analysis has also demonstrated the benefits of using graph coloring for resources coordination.

\section{REFERENCES}

[1] T.R. Jensen and B. Toft; "Graph Coloring Problem", Wiley InterScience, 1995

[2] S. Ramanathan and E. L. Lloyd, Scheduling algorithms for multihop radio networks, IEEE/ACM Trans. Networking 1 (April 1993) 166-172.

[3] S. Ramanathan, "A unified framework and algorithm for channel assignment in wireless networks", Wireless Networks, v.5 n.2, p.81-94, March 1999

[4] S. Even, O. Goldreich, S. Moran and P. Tong, On the NPcompleteness of certain network testing problems, Networks 14 (1984) 1-24

[5] A. Ephremides and T. V. Truong, Scheduling broadcasts in multihop radio networks, IEEE Trans. Communications 38 (April 1990)

[6] Arunabha Sen, Mark L. Huson, A new model for scheduling packet radio networks, Wireless Networks, v.3 n.1, p.71-82, March 1997

[7] Wagon, S. "An April Fool's Hoax." Mathematica in Educ. Res. 7, 46-52, 1998

[8] J. A. Bondy and U. S. R. Murty; "Graph Theory with Application", American Elsevier Publishing Co. Inc., New York, 1976

[9] A. Sen and M.L. Huson, A new model for scheduling packet radio networks, in: Proceedings of INFOCOM (1996) pp. 11161124 


\section{Proceedings}

\section{IEEE 8th International Conference on Computer and Information Technology}

\section{CIT 2008}

8-11 July $2008 \cdot$ Sydney, Australia

\section{Editors}

Qiang Wu, Xiangjian He, Quang Vinh Nguyen, Wenjing Jia and Maolin Huang

\section{Organized by}

\section{CIT Organizing Committee}

In cooperation with

IEEE Computer Society

IEEE Technical Committee on Scalable Computing University of Technology, Sydney

Research Institute for Information and Communication Technology, Korea University, Korea

BK 21 Information Technology Division, Korea University, Korea ARC Research Network in Enterprise Information Infrastructure (EII), Australia Federation of Chinese Scholars in Australia (FOCSA), Australia Australian Chinese ICT Professional Society, Australia 


\section{MESSAGE FROM CHAIRS}

On behalf of the Organizing Committee and the Technical Program Committee, it is our great pleasure to welcome you to Sydney and the IEEE 8th International Conference on Computer and Information Technology 2008 (CIT2008).

As an IEEE CS fully sponsored conference, International Conference on Computer and Information Technology (CIT) has become a major platform for researchers and industry practitioners from different fields of computer and information technology. Each year, CIT attendees appreciate and benefit from multidisciplinary exchanges in computer and information technology. CIT has attracted many high quality research papers which highlight foundational work that strives to push beyond limits of existing computer technologies, including experimental efforts, innovative systems, and investigations that identify weaknesses in existing IT services.

The topics of CIT2008 include:

- Database and Data Mining

- High Performance Computing

- Multimedia and Computer Graphics

- Ubiquitous and Sensor Networks

- Information Systems

- Service Oriented Computing
- Internet and Web Applications

- Networking and Mobile Computing

- Image Processing, Computer Vision and Video Surveillance

- VLSI and Computer Systems

- Software Engineering

This year, the number of papers submitted to CIT2008 has overwhelmed those of previous editions. CIT2008 received more than 550 submissions coming from various countries and selected about $150(27 \%)$ as CIT2008 regular papers to be included in the CIT2008 proceedings. The revision process for all papers has been rigorous and thorough, including peer-reviewing from at least two independent and qualified reviewers, and reviewing from the local organization committee. The accepted papers for this volume of proceedings are expected to be of very high quality. We would like to express our sincere gratitude to all the reviewers, members of the Program Committee and the organization committee who have provided CIT2008 with such a qualified technical program. We also would like to express our special appreciation to the following organizers of various invited sessions of CIT2008, Dr. Liang Wang, Dr. Ming Li, Dr. Xin Geng, Dr. Jiali Cui, Dr. Joan Lu, Dr. Fahim Akhter and Dr. Huwida Said.

We deeply thank all the authors, attendees, sponsors, organizers and all others who have been involved in organizing this event at any level for their invaluable support. We hope that you will enjoy the conference in all its aspects so that we will be able to count on your interest and support also in the future.

Xiangjian (Sean) He
General Chair, CIT2008
Qiang Wu

Program Chair, CIT2008 


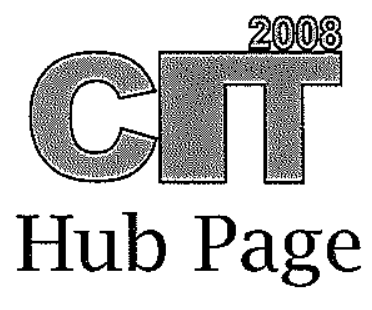

\section{IEEE $8^{\text {th }}$ International Conference on Computer and Information Technology}

8-11 July 2008 - Sydney, Australia

\begin{tabular}{|c|c|}
\hline Welcome Page & * Session List \\
\hline Welcome from Chairs & Table of Contents \\
\hline Organizing Committee & Author Index: Brief or Detailed \\
\hline Program Committee & Manuscripts \\
\hline Additional Reviewers & Sponsors \\
\hline
\end{tabular}

Proceedings of the 2008 IFFE 8 th International Conference on Computer and Information Technology. IFFE Cataiog Number CFP08355-CDR, ISBN 978-1-4244-2358-3, Library of Congress 2008902859. Copyright () 2008 IEEE. Personal use of this material is permitted. However, permission to reprint/republish this material for advertising or promotional purposes or for creating new collective works for resale or redistribution to servers or lists, or to reuse any copyrighted component of this work in other works must be obtained from the IFFE. For technical support please contact Causal Productions (info@causalproductions.com).

Legend: View Manuscript

[Search]

Authorized licensed use limited to: University of Technology Sydney. Downloaded on March 13, 2009 at 01:09 from IEEE Xplore. Restrictions apply. 